\title{
Stroke Early Supported Discharge (ESD): The impact of patients' characteristics and clinical profile on rehabilitation goal attainment and clinical outcomes
}

Norfolk Community W/HS Dr Ehab Georgy, MCSP, BSc, MSc, PhD Health and Care

On behalf of the Suffolk Stroke ESD Team, UK

\section{Introduction}

$\ddot{\gamma}$ ESD services were introduced to provide a specialist stroke therapy input, whilst reducing cost of acute care.

$\ddot{\gamma}$ ESD resulted in better outcomes.

$\ddot{\gamma}$ A consensus is lacking regarding specific ESD patient characteristics \& clinical profile.
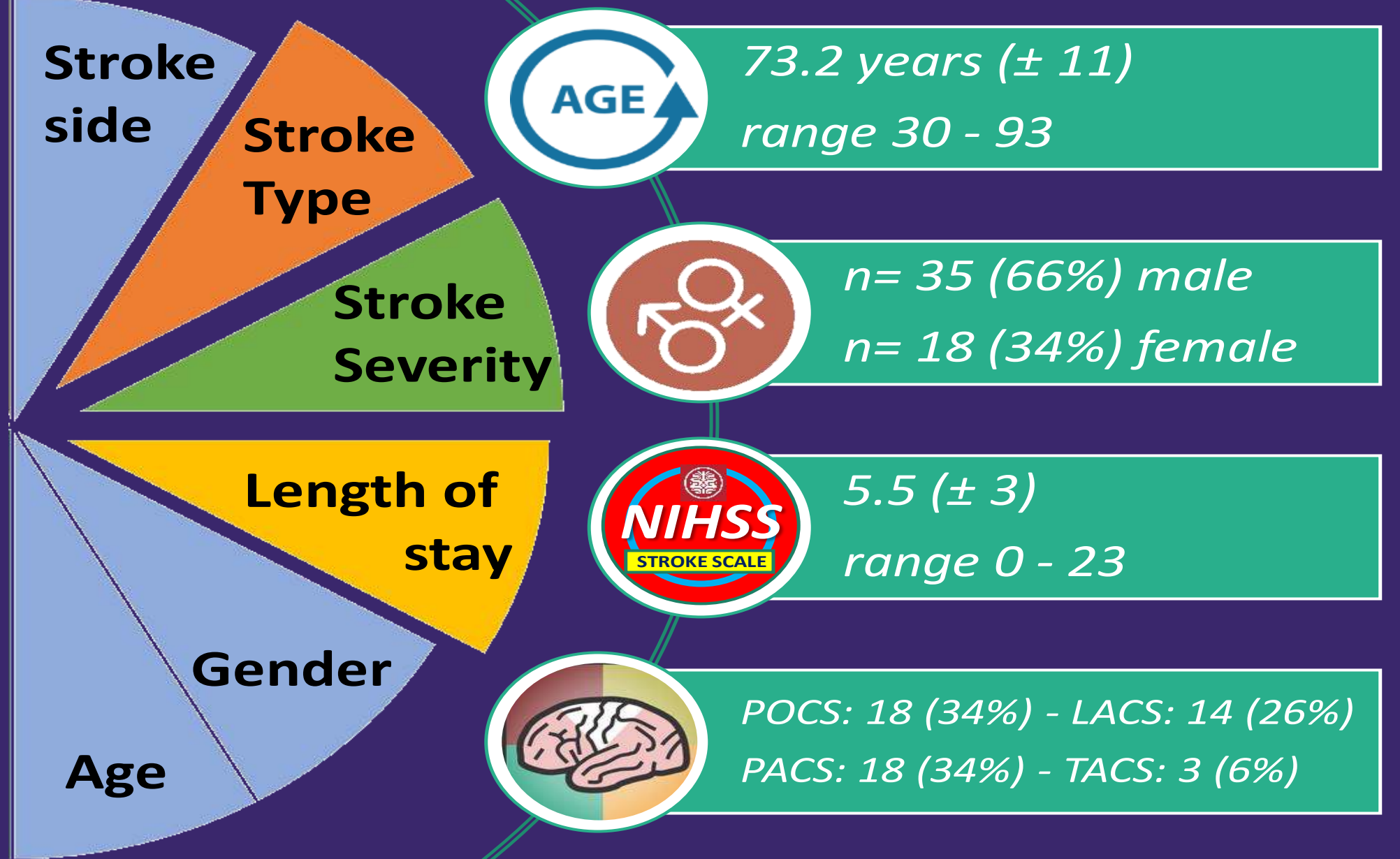

\begin{tabular}{|c|c|c|c|c|c|}
\hline $\begin{array}{l}\text { Goal } \\
\text { attainment }\end{array}$ & n (\%) & \multicolumn{2}{|c|}{$\begin{array}{c}\text { baseline } \\
\text { score }\end{array}$} & $\begin{array}{l}\text { final } \\
\text { score }\end{array}$ & $\begin{array}{c}\text { score } \\
\text { change }\end{array}$ \\
\hline not achieved & $15(28 \%)$ & \multicolumn{2}{|c|}{33.0} & 45.1 & $12.1(37 \%)$ \\
\hline fully achieved & $26(49 \%)$ & \multicolumn{2}{|c|}{37.7} & 50 & $12.3(32 \%)$ \\
\hline \multirow[t]{2}{*}{ higher achieving } & $12(23 \%)$ & & 37.0 & 58.2 & $21.2(57 \%)$ \\
\hline & \multicolumn{2}{|c|}{ Not achieved } & \multicolumn{2}{|c|}{ Fully achieved } & Highly achieved \\
\hline $\begin{array}{c}\text { Discharge } \\
\text { destination } \\
\end{array}$ & \multicolumn{2}{|c|}{$\begin{array}{c}\text { Home } 93 \% \\
\text { Care Home } 7 \%\end{array}$} & \multicolumn{2}{|c|}{$\begin{array}{c}\text { Home } 100 \% \\
\text { Care Home 0\% }\end{array}$} & $\begin{array}{c}\text { Home } 100 \% \\
\text { Care Home 0\% }\end{array}$ \\
\hline Onward referrals & \multicolumn{2}{|l|}{$80 \%$} & \multicolumn{2}{|c|}{$23 \%$} & $42 \%$ \\
\hline
\end{tabular}

\section{Conclusion}

- Specific patient cohort seem to benefit the most from ESD. - Specific clinical characteristics and disease profile correlate with functional outcomes \& could affect recovery \& goal attainment post-stroke.

Future research is needed to establish specific ESD criteria to stratify stroke patients \& identify those with best chance of optimal functional outcome.

\section{Objectives}

To provide clinical data to support the development of ESD patient profile (demographics \& clinical) $\&$ establish the impact on clinical outcomes in terms of functional recovery and goal attainment.

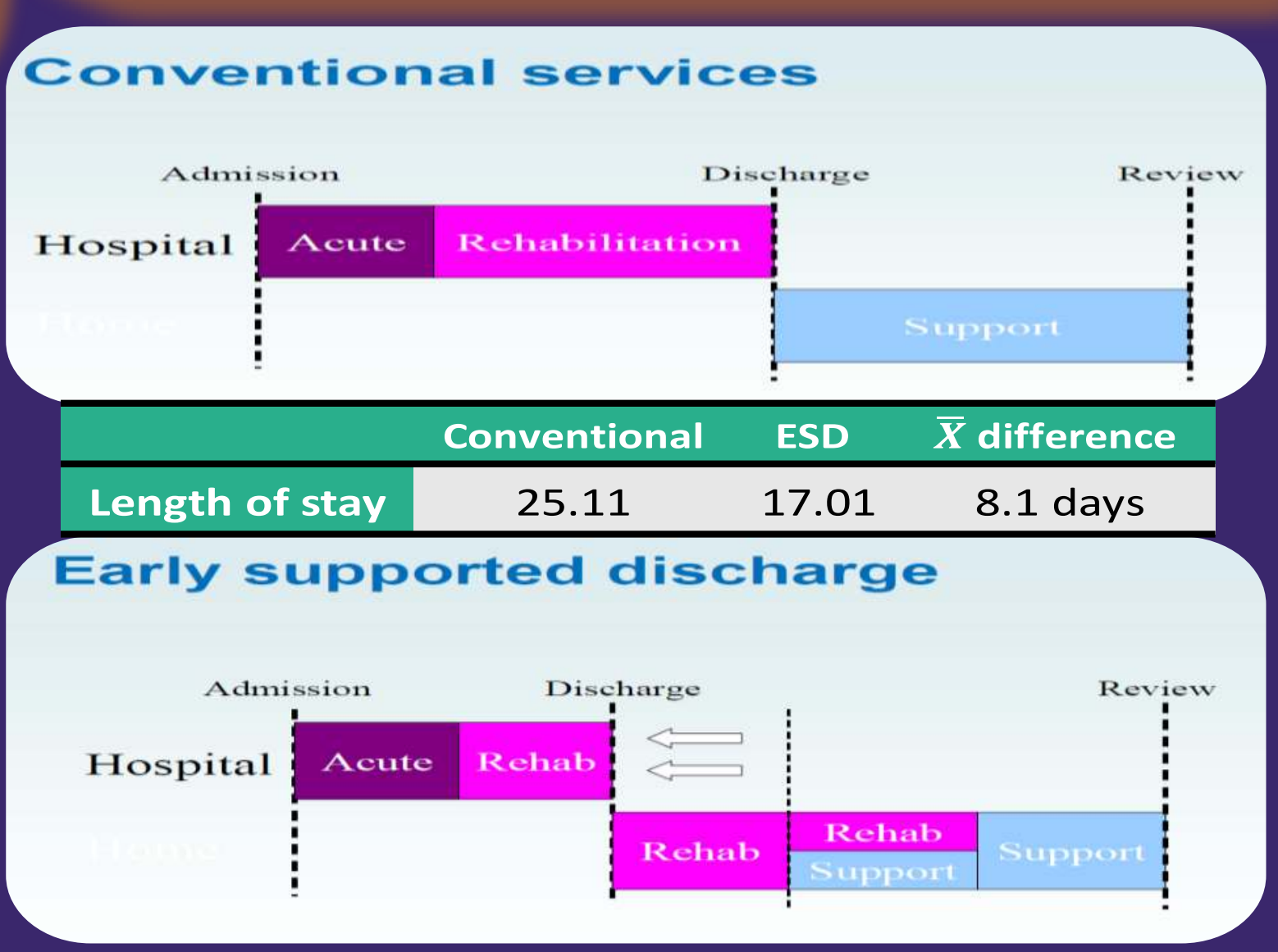

$\mathrm{mRs}$

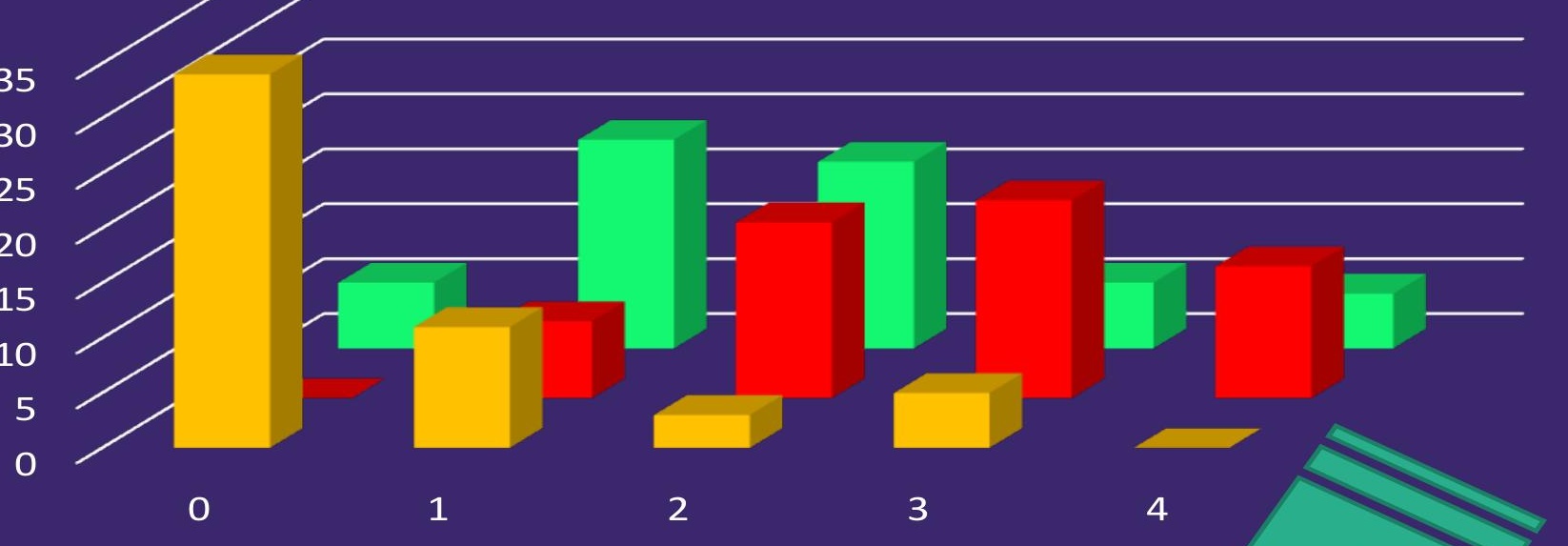

Baseline Pre-ESD $\square$ Post-ESD

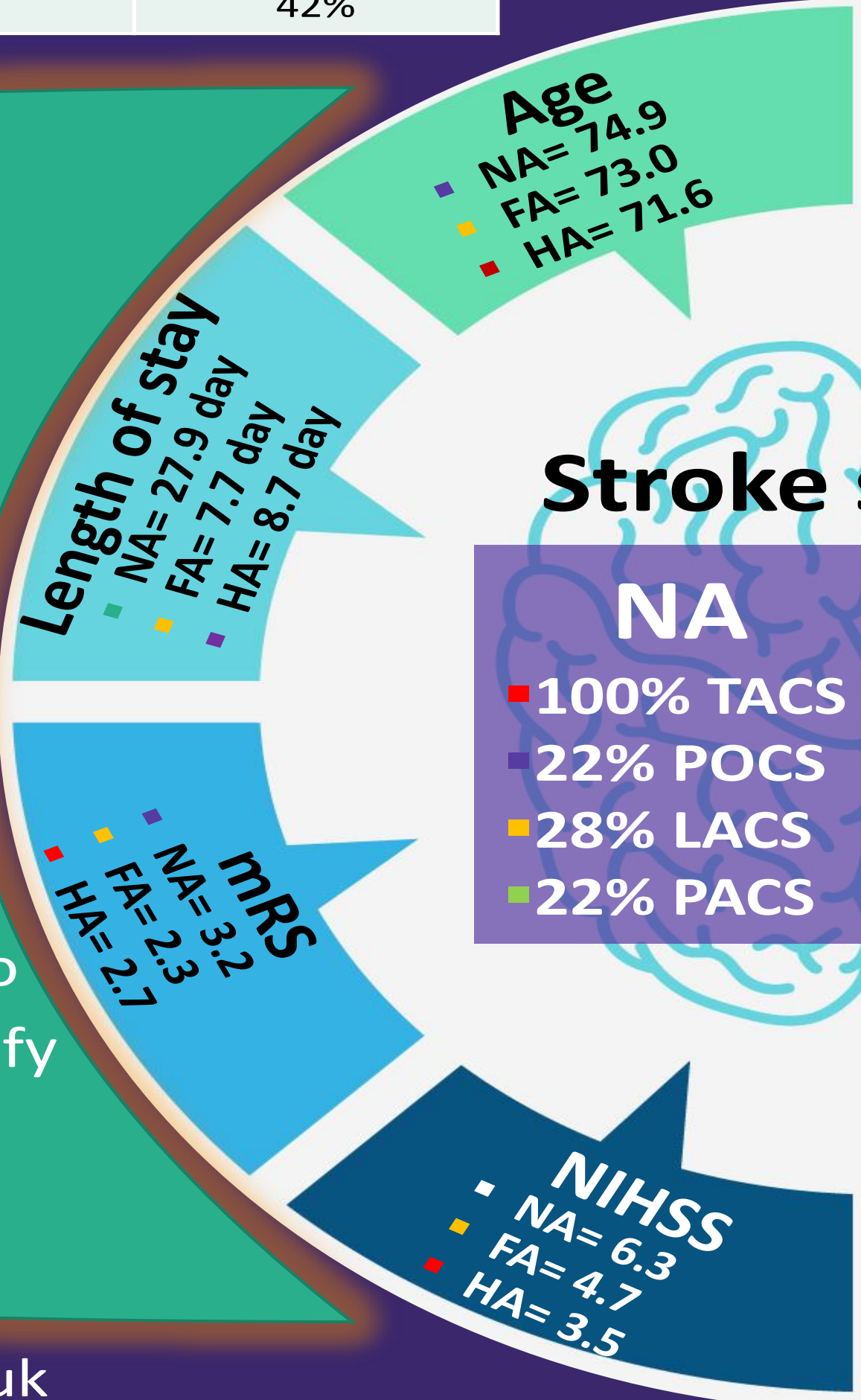

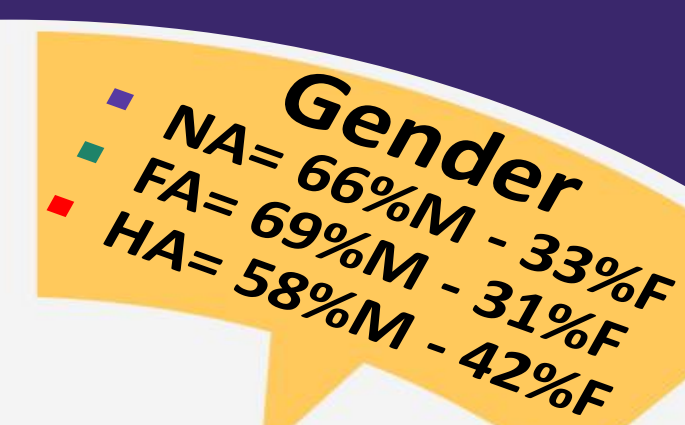

